\title{
An alternative proof of a characterization of the TTC Mechanism
}

\author{
Jay Sethuraman*
}

April 2015; October 2015; November 2015

\begin{abstract}
For the problem of reallocating indivisible objects among a set of agents with strict preferences, Ma characterized the Top-Trading Cycles (TTC) mechanism using the properties of Pareto efficiency, individual rationality, and strategy-proofness. A simpler, direct proof of this result is presented here.
\end{abstract}

KEYWORDS. housing market; core mechanism; top-trading cycles

\section{Introduction}

We consider the standard housing market model first studied by Shapley and Scarf [7]. There are $n$ agents $N=\{1, \ldots, n\}$. Each agent is initially endowed with an object and has strict preferences over all the objects, including his endowment. To avoid cumbersome notation we call agent $i$ 's endowment as object $i$. We denote agent $i$ 's preference ordering by $\geq_{i}$, and $\left(\geq_{1}, \geq_{2}, \ldots, \geq_{n}\right)$ denotes the preference profile for all agents. We write $a>_{i} b$ to indicate that agent $i$ strictly prefers object $a$ to object $b$, and $a \geq_{i} b$ to indicate that $i$ weakly prefers $a$ to $b$. An allocation is a re-distribution of the objects in which each agent obtains exactly one object: thus any allocation can be identified with a permutation $\mu$ of the objects, where agent $i$ is allocated object $\mu_{i}$. An allocation $\mu$ is Pareto-efficient if there is no allocation $\mu^{\prime}$ such that $\mu_{i}^{\prime} \geq_{i} \mu_{i}$ for all $i \in N$ with at least one of the inequalities holding strictly; that is, we cannot improve agent $i$ 's allocation without making some other agent worse-off. An allocation $\mu$ is individually-rational if each agent $i$ (weakly) prefers $\mu_{i}$ to object $i$. A mechanism is a function that maps each preference profile to an allocation. A mechanism is Pareto-efficient (individually rational) if it always finds an allocation that is Pareto efficient (individually rational) on any preference profile. A mechanism is strategy-proof if reporting her preference ordering truthfully is a weakly dominant strategy for each agent: that is, each agent weakly prefers the object she obtains when she reports her true preference ordering to the object she is assigned under any other report, regardless of what the other agents report.

For any preference profile $P$, define the size $s(P)$ of $P$ as the total number of objects that the agents find acceptable in $P$. That is, $s(P)=\sum_{i \in N}\left|\left\{a \mid a \geq_{i} i\right\}\right|$. Note that the $s(P)$ is at least $n$, and is equal to $n$ if and only if each agent's most-preferred object is her endowment.

\section{The Main Result}

Theorem 1. For the housing market problem, there is at most one strategy-proof mechanism that is individually rational and Pareto efficient.

${ }^{*}$ IEOR Department, Columbia University, New York, NY; jay@ieor.columbia.edu. 
Proof. Let $\Pi$ and $\Psi$ be distinct strategy-proof, individually rational and Pareto efficient mechanisms. For any profile $P$, let $\pi_{i}^{P}$ be the object allocated to agent $i$ by the mechanism $\Pi$, and let $\psi_{i}^{P}$ be the object allocated to agent $i$ by the mechanism $\Psi$. As $\Pi \neq \Psi$, there is some profile for which these two mechanisms find different allocations for some agent. Among all such profiles, let $P$ be one that has the smallest size. Let $A$ be the set of agents who are assigned different objects in $\Pi$ and $\Psi$ for the profile $P$. By assumption, $A \neq \emptyset$. We claim that each agent in $A$ has exactly two acceptable objects in $P$.

Suppose not. Pick an agent $i \in A$ with more than two acceptable objects, and suppose $i$ strictly prefers $\pi_{i}^{P}$ to $\psi_{i}^{P}$. Modify the preference ordering of agent $i$ by removing all objects other than $\pi_{i}^{P}$ and (her endowment) object $i$; all other agents hold the same preferences. Call the resulting preference profile $Q$. By strategy-proofness of $\Pi$ and $\Psi, \psi_{i}^{Q} \neq \pi_{i}^{Q}=\pi_{i}^{P}$; thus, agent $i$ should retain her endowment in $\Psi$. In particular, $Q$ is a profile in which the two mechanisms disagree, and has a smaller size, contradicting our choice of $P$.

Let $A_{\Pi}$ be the set of agents in $A$ who strictly prefer their assigned object in $\Pi$ to that in $\Psi$; the set $A_{\Psi}$ is defined similarly. Clearly, $A_{\Pi} \cap A_{\Psi}=\emptyset$, and $A_{\Pi} \cup A_{\Psi}=A$; thus at least one of $A_{\Pi}, A_{\Psi}$ is non-empty. Suppose $A_{\Pi} \neq \emptyset$. Note that $\Pi$ assigns to each agent in $A_{\Pi}$ an object she strictly prefers to her endowment, whereas $\Psi$ assigns each such agent her endowment. Similarly, $\Psi$ assigns to each agent in $A_{\Psi}$ an object she strictly prefers to her endowment, whereas $\Pi$ assigns each such agent her endowment. Now, pick $k \in A_{\Pi}$. Observe that object $k$ (the endowment of agent $k$ ) must be assigned by mechanism $\Pi$ to some other agent in $A_{\Pi}$ - this is because agents in $A_{\Psi}$ retain their endowments in $\Pi$ and so cannot be assigned object $k$; agents outside of $A$ have the same assignment in both $\Pi$ and $\Psi$ and so cannot be assigned object $k$ (the assumption of strict preferences is crucial here). Thus, both $\Pi$ and $\Psi$ assign the same set of objects to the agents in $A_{\Pi}$, each of whom prefers her assignment in $\Pi$ to that in $\Psi$. So, the assignment obtained by giving object $\pi_{i}^{P}$ to each agent $i \in A_{\Pi}$, and giving object $\psi_{i}^{P}$ to each agent $i \notin A_{\Pi}$ Pareto dominates the assignment found by $\Psi$ at the profile $P$, a contradiction.

It is easy to verify that the TTC mechanism is strategy-proof, Pareto efficient, and individually rational [7, 5]. An immediate consequence of Theorem 1 is that the TTC mechanism is the only mechanism that is Pareto efficient, individually rational, and strategy-proof, a result first proved by Ma [4].

\section{Discussion}

It is instructive to compare the proof presented here with the original proof of Ma [4] and a recent simplification of that proof due to Anno [1]. The original proof of Ma is long, but insightful; Anno's recent proof is substantially simpler, and follows the same general structure. Both proofs begin with the observation that the TTC mechanism, which is Pareto efficient, individually rational, and strategy-proof, finds an allocation in the core of the associated cooperative game. Then they establish the following important fact $(*)$ : in any other mechanism $\Gamma$ that is strategy-proof, individually rational, and Pareto efficient, there must be an agent who trades his endowment, but strictly prefers his allocation in the TTC mechanism to his allocation under $\Gamma$. If one eliminates every object other than his TTC-allocation and his endowment from his preference list, his allocation in the TTC mechanism should remain the same, but his allocation in $\Gamma$ must be his endowment (by strategy-proofness); by fact $(*)$, there must now be a different agent who trades, but does worse in $\Gamma$ than in TTC. As there are only finitely many agents, this process must stop, leading to a contradiction. The original proof of $\mathrm{Ma}$, and a later proof by Svensson [8], required a rather tedious "preference replacement" step; Anno's remarkable simplification avoids such a step and establishes the result in a simpler way. Like Anno, we 
exploit strategy-proofness to argue that agents who have different assignments in the two mechanisms have exactly two acceptable objects: in our proof, this is a consequence of examining a smallest-size profile at which the mechanisms differ, whereas in Anno's case this is iteratively established explicitly. The key difference however is that Theorem 1 does not assume or exploit the existence of a core mechanism; in fact, it does not even assert the existence of a mechanism that satisfies the stated properties. Instead, it exploits the assumption that agents have strict preferences to argue that one cannot have distinct mechanisms satisfying the stated properties.

It is useful to see where the proof breaks down when agents have indifferences in preferences. The natural analog of Theorem 1 is the following statement: If $\Pi$ and $\Psi$ are distinct Pareto efficient, individually rational and strategy-proof mechanisms, then each agent $i$ is indifferent between $\Pi_{i}^{P}$ and $\Psi_{i}^{P}$ at every preference profile $P$. Unfortunately, this statement is false as shown by the following simple three agent example: suppose agent 1 is indifferent between objects 2 and 3 ; whereas agents 2 and 3 both strictly prefer object 1 to their endowment, and find each other's endowment unacceptable. Pareto efficiency and individual rationality imply that agent 1 must trade with agent 2 or agent 3 , but not both; and each of these outcomes could be achieved by a strategy-proof mechanism [6]. But agent 2 is not indifferent between his allocation in these two outcomes. The key step where the proof fails is when we argue that the endowments of the agents in $A_{\Pi}$ should be allocated to other agents in $A_{\Pi}$ - this is true only when agents who are outside of $A$ have identical allocations in the mechanisms $\Pi$ and $\Psi$; in the case of indifferences in preferences, the only conclusion we can draw is that agents outside of $A$ are indifferent between their allocations in $\Pi$ and $\Psi$ (like agent 1 is in the two allocations in our example). Indeed in our example, the endowment of the agent in $A_{\Pi}$ is allocated to agent 1 who is not in $A$, and so one cannot conclude Pareto dominance.

Finally, we mention a related strand in the literature on allocating indivisible goods with indifferences that does generalize Ma's characterization result: if $\Pi$ and $\Psi$ are distinct Pareto efficient, individually rational and strategy-proof mechanisms, then each agent $i$ is indifferent between $\Pi_{i}^{P}$ and $\Psi_{i}^{P}$ at every preference profile $P$ with a non-empty core. The papers of Wako [10], Sönmez [9], and Ehlers [3] contain different versions of this result as well as extensions and generalizations; the recent paper of Anno [2] contain a simpler proof for an important special case.

\section{Acknowledgments}

I thank Tayfun Sönmez and Utku Ünver for raising this question during a seminar at Boston college. I thank Daniela Saban and Chun Ye for hearing various versions of this argument, and for their comments on an earlier draft of this proof. I thank the associate editor and anonymous referees for bringing references [1] and [2] to my attention as well as for their comments on an earlier version of this paper. This research was supported by NSF grant CMMI 1201045.

\section{References}

[1] AnNo, H. 2015. A short proof for the characterization of the core in housing markets. Economics Letters 126, 1, 66 - 67.

[2] Anno, H. 2014. A short proof for the essentially single-valued core theorem. Manuscript.

[3] Ehlers, L. 2014. Strategy-proofness and essentially single-valued cores revisited. Manuscript. 
[4] MA, J. 1994. Strategy-proofness and the strict core in a market with indivisibilities. International Journal of Game Theory 23, 1, 75-83.

[5] Roth, A. 1982. Incentive compatibility in a market with indivisible goods. Economics Letters 9, 2, $127-132$.

[6] Saban, D. And Sethuraman, J. 2013. House Allocation with Indifferences: A generalization and a unified view. Proceedings of the 14th annual ACM EC conference, 803-820.

[7] Shapley, L. And Scarf, H. 1974. On cores and indivisibility. Journal of Mathematical Economics 1, 1, 23 - 37.

[8] Svensson, L.-G. 1999. Strategy-proof allocation of indivisible goods Social Choice and Welfare 16, $557-567$.

[9] Sönmez, T. 1999. Strategy-proofness and Essentially Single-valued Cores. Econometrica $67,3,677-689$.

[10] WaKo, J. 1991. Some properties of weak domination in an exchange market with indivisible goods Economic Studies Quarterly 42,, 303 - 314. 